\title{
Interactive Constraint-Based Sequential Pattern Mining *
}

\author{
Marek Wojciechowski \\ Poznan University of Technology \\ Institute of Computing Science \\ ul. Piotrowo 3a, 60-965 Poznan, Poland \\ Marek.Wojciechowski@cs.put.poznan.pl
}

\begin{abstract}
Data mining is an interactive and iterative process. It is very likely that a user will execute a series of similar queries differing in pattern constraints and mining parameters, before he or she gets satisfying results. Unfortunately, data mining algorithms currently available suffer from long processing times, which is unacceptable in case of interactive mining. In this paper we discuss efficient processing of sequential pattern queries utilizing cached results of other sequential pattern queries. We analyze differences between sequential pattern queries and propose algorithms that in many cases can be used instead of time-consuming mining algorithms.
\end{abstract}

\section{Introduction}

Data mining aims at discovery of useful patterns from large databases or warehouses. One of the most popular data mining methods is sequential pattern discovery introduced in [2]. Informally, sequential patterns are the most frequently occurring subsequences in sequences of sets of items. The initial formulation of the problem was significantly extended in [10], where a taxonomy on items was added to support discovery of so called generalized sequential patterns, and three time constraints (min-gap, max-gap, and time window) were introduced to be used when checking if a given source sequence contains a given pattern. For that extended problem formulation, an efficient algorithm called GSP was proposed. Applications of sequential patterns include analysis of telecommunication systems, discovering frequent buying patterns, analysis of patients' medical records, etc.

From a user's point of view, data mining can be seen as an interactive and iterative process of advanced querying: a user specifies the source dataset and the requested class of patterns, the system chooses the appropriate data mining algorithm and returns discovered patterns to the user [4][6]. A user interacting with a data mining system has to specify several constraints on patterns to be discovered. However, usually it is not trivial to find a set of constraints leading

\footnotetext{
* This work was partially supported by the grant no. KBN 43-1309 from the State
} Committee for Scientific Research (KBN), Poland. 
to the satisfying set of patterns. Thus, users are very likely to execute a series of similar data mining queries before they find what they need. Unfortunately, data mining algorithms require long processing times, which makes such interaction difficult.

In this paper, we discuss efficient sequential pattern discovery in the presence of materialized results of previous sequential pattern queries. We claim that a data mining system should exploit the fact that a user is very likely to execute a number of similar sequential pattern queries during a single session. We propose caching results of mining queries by materializing their results on disk (we assume that a data mining system is going to be assigned a certain amount of disk space for that purpose). It is obvious that materialized results of a query can be used to answer an identical query, therefore we concentrate on processing queries different from those whose results are available. The possibility of answering a query using known results of another query depends on the differences between the two queries. Our goal is to provide criteria for determining if cached results of a given query can be used to answer the current query without running a complete mining algorithm, and introduce efficient sequential pattern query processing algorithms exploiting materialized patterns.

Exploiting cached results of previous mining queries has been studied in the context of association rules [3][7]. However, direct application of methods and techniques introduced for association rules to sequential pattern discovery problem is not possible since different types of constraints are available in the two problems. Nevertheless, it seems that the general ideas should stay unchanged.

In has been observed [3] that the three particularly interesting relationships between two mining queries $D M Q_{1}$ and $D M Q_{2}$ extracting patterns from the same data are equivalence, inclusion, and dominance. The three relationships are interesting since they represent situations, where one data mining query can be efficiently answered using the results of another query. Differences between mining queries leading to these relationships were analyzed only in the context of association rules. In this paper we present analogous analysis concerning sequential patterns. Thus, most of our work can be regarded as the extension of the approach from [3] into sequential pattern discovery.

\subsection{Sequential Patterns}

Let $L=l_{1}, l_{2}, \ldots, l_{m}$ be a set of literals called items. An itemset is a non-empty set of items. A sequence is an ordered list of itemsets and is denoted as < $X_{1} X_{2} \ldots X_{n}>$, where $X_{i}$ is an itemset $\left(X_{i} \subseteq L\right) . X_{i}$ is called an element of the sequence. The size of a sequence is the number of items in the sequence. The length of a sequence is the number of elements in the sequence. Let $D$ be a set of variable length sequences (called data-sequences), where for each sequence $S=<X_{1} X_{2} \ldots X_{n}>$, a timestamp is associated with each $X_{i}$.

With no time constraints we say that a sequence $X=<X_{1} X_{2} \ldots X_{n}>$ is contained in a data-sequence $Y=<Y_{1} Y_{2} \ldots Y_{m}>$ if there exist integers $i_{1}<i_{2}<$ $\ldots<i_{n}$ such that $X_{1} \subseteq Y_{i_{1}}, X_{2} \subseteq Y_{i_{2}}, \ldots, X_{n} \subseteq Y_{i_{n}}$. We call $<Y_{i_{1}} Y_{i_{2}} \ldots Y_{i_{n}}>$ an occurrence of $X$ in $Y$. We consider the following user-specified time constraints 
while looking for occurrences of a given sequence: minimal and maximal gap allowed between consecutive elements of an occurrence of the sequence (called min-gap and max-gap), and time window that allows a group of consecutive elements of a data-sequence to be merged and treated as a single element as long as their timestamps are within the user-specified window-size.

The support of a sequence $\left\langle X_{1} X_{2} \ldots X_{n}\right\rangle$ in $D$ is the fraction of datasequences in $D$ that contain the sequence. A sequential pattern is a sequence whose support in $D$ is above the user-specified threshold.

\subsection{Relationships between Results of Data Mining Queries}

Two data mining queries are equivalent if for all datasets they both return the same set of patterns and the values of statistical significance measures (e.g. support) for each pattern are the same in both cases. A data mining query $D M Q_{1}$ includes a data mining query $D M Q_{2}$ if for all datasets each pattern in the results of $D M Q_{2}$ is also returned by $D M Q_{1}$ with the same values of the statistical significance measures. A data mining query $D M Q_{1}$ dominates a data mining query $D M Q_{2}$ if for all datasets each pattern in the results of $D M Q_{2}$ is also returned by $D M Q_{1}$, and for each pattern returned by both queries its values of the statistical significance measures evaluated by $D M Q_{1}$ are not less than is case of $D M Q_{2}$. Equivalence is a particular case of inclusion, and inclusion is a particular case of dominance. Equivalence, inclusion, and dominance meet the transitivity property.

If for a given query, results of a query equivalent to it, including it, or dominating it are available, the query can be answered without running a costly mining algorithm. In case of equivalence no processing is necessary, since the queries have the same results. In case of inclusion, one scan of the materialized query results is necessary to filter out patterns that do not satisfy constraints of the included query. In case of dominance, one verifying scan of the source dataset is necessary to evaluate the statistical significance of materialized patterns (filtering out the patterns that do not satisfy constraints of the dominated query is also required).

\subsection{Related Work}

To facilitate interactive and iterative pattern discovery, [8] proposed to materialize patterns discovered with the least restrictive selection criteria, and answer incoming queries by filtering the materialized pattern collection. This approach is not a perfect solution of the problem since pattern mining with very low minimum support thresholds might lead to collections of frequent patterns even larger than the original database. Moreover, restricting certain constraints (e.g. time constraints in the context of sequential pattern mining) not only makes some patterns infrequent but also changes the support of patterns that remain frequent.

Much more reasonable and flexible solutions supporting interactive and iterative mining were presented in [7], in the context of association rules. The 
solutions presented there consisted in caching results of mining queries. In the approach, materialization of frequent itemsets instead of rules was proposed. However, in some cases it was required to materialize also some of the infrequent itemsets.

Most of the research on sequential patterns focused on introducing new algorithms, more efficient than GSP (e.g. [5][9]). However, the novel methods do not handle time constraints and taxonomies. Thus, GSP still remains the most general sequential pattern discovery algorithm and the reference point for new methods and techniques.

\subsection{Organization of the Paper}

The paper is organized as follows. Section 2 presents constraints that can be specified in sequential pattern mining. In Sect.3, relationships between sequential pattern queries are discussed. Section 4 contains efficient sequential pattern query processing algorithms. Experimental results concerning the proposed algorithms are presented in Sect.5. We conclude with a summary in Sect.6.

\section{Constraint-Based Sequential Pattern Mining}

In constraint-based sequential pattern mining, we identify the following classes of constraints: database constraints, pattern constraints, and time constraints. Database constraints are used to specify the source dataset. Pattern constraints specify which patterns are interesting and should be returned by the query. Finally, time constraints influence the process of checking whether a given datasequence contains a given pattern.

The basic formulation of the sequential pattern discovery problem introduces three time constraints: max-gap, min-gap, and time window, and assumes only one pattern constraint (expressed by means of the minimum support threshold). We model pattern constraints as complex Boolean predicates having the form of a conjunction of basic Boolean predicates on patterns presented below:

- $\pi$ (SPG, $\alpha$, pattern $)$ - true if pattern support is greater than $\alpha$, false otherwise;

- $\pi$ (SL, $\alpha$, pattern $)$ - true if pattern size is less than $\alpha$, false otherwise;

$-\pi$ (SG, $\alpha$, pattern) - true if pattern size is greater than $\alpha$, false otherwise;

$-\pi(\mathbf{L L}, \alpha$, pattern $)$ - true if pattern length is less than $\alpha$, false otherwise;

- $\pi(\mathbf{L G}, \alpha$, pattern $)$ - true if pattern length is greater than $\alpha$, false otherwise;

$-\pi(\mathbf{C}, \beta$, pattern $)$ - true if $\beta$ is a subsequence of the pattern, false otherwise;

$-\pi(\mathbf{N C}, \beta$, pattern $)$ - true if $\beta$ is not a subsequence of the pattern, false otherwise.

We believe that the above list of predicates is sufficient to allow users to express their pattern selection criteria. For simplicity's sake, in length and size predicates we consider only sharp inequalities. 


\section{Relationships between Sequential Pattern Queries}

Inclusion and dominance relationships between two data mining queries are defined for queries operating on the same dataset. Therefore, analyzing differences between sequential pattern queries, we consider only differences in time and pattern constraints.

Definition 1. Given two basic Boolean pattern predicates $b_{1}$ and $b_{2}$, we say that $b_{2}$ is stronger than $b_{1}$ if one of the following conditions holds:

1. $b_{1}=\pi\left(\mathbf{S P G}, \alpha_{1}\right.$, pattern) and $b_{2}=\pi\left(\mathbf{S P G}, \alpha_{2}\right.$, pattern), where $\alpha_{2}>\alpha_{1}$,

2. $b_{1}=\pi\left(\mathbf{S G}, \alpha_{1}\right.$, pattern) and $b_{2}=\pi\left(\mathbf{S G}, \alpha_{2}\right.$, pattern $)$, where $\alpha_{2}>\alpha_{1}$,

3. $b_{1}=\pi\left(\mathbf{S L}, \alpha_{1}\right.$, pattern) and $b_{2}=\pi\left(\mathbf{S L}, \alpha_{2}\right.$, pattern), where $\alpha_{2}<\alpha_{1}$,

4. $b_{1}=\pi\left(\mathbf{L G}, \alpha_{1}\right.$, pattern) and $b_{2}=\pi\left(\mathbf{L G}, \alpha_{2}\right.$, pattern $)$, where $\alpha_{2}>\alpha_{1}$,

5. $b_{1}=\pi\left(\mathbf{L L}, \alpha_{1}\right.$, pattern) and $b_{2}=\pi\left(\mathbf{L L}, \alpha_{2}\right.$, pattern $)$, where $\alpha_{2}<\alpha_{1}$,

6. $b_{1}=\pi\left(\mathbf{C}, \beta_{1}\right.$, pattern $)$ and $b_{2}=\pi\left(\mathbf{C}, \beta_{2}\right.$, pattern $)$, where a pattern $\beta_{1}$ is a subsequence of the pattern $\beta_{2}$ and the size of $\beta_{1}$ is less than the size of $\beta_{2}$,

7. $b_{1}=\pi$ (NC, $\beta_{1}$, pattern) and $b_{2}=\pi\left(\mathbf{N C}, \beta_{2}\right.$, pattern $)$, where pattern $\beta_{2}$ is a subsequence of the pattern $\beta_{1}$ and the size of $\beta_{2}$ is less than the size of $\beta_{1}$.

Definition 2. We say that a data mining query $D M Q_{2}$ extends pattern constraints of a data mining query $D M Q_{1}$ if any of the following conditions holds:

1. Pattern constraints of $D M Q_{1}$ have a form of a conjunction of $n$ basic Boolean pattern predicates, pattern constraints of $D M Q_{2}$ have a form of a conjunction of $n+1$ basic Boolean pattern predicates $(n \geq 0)$, and each basic Boolean pattern predicates in $D M Q_{1}$ also appears in $D M Q_{2}$;

2. $D M Q_{1}$ and $D M Q_{2}$ have pattern constraints $p_{1}$ and $p_{2}$ respectively, where $p_{1}$ and $p_{2}$ are conjunctions of $n$ basic Boolean pattern predicates $(n \geq 1)$, $p_{1}=p \wedge b_{1}, p_{2}=p \wedge b_{2}$ ( $p$ is a conjunction of $n-1$ basic Boolean pattern predicates), and $b_{2}$ is stronger than $b_{1}$;

3. It is possible to formulate a data mining query $D M Q_{3}$ such that $D M Q_{2}$ extends pattern constraints of $D M Q_{3}$ and $D M Q_{3}$ extends pattern constraints of $D M Q_{1}$. (The relationship of extending pattern constraints is transitive.)

In other words, a data mining query $D M Q_{2}$ extends pattern constraints of a data mining query $D M Q_{1}$ if pattern constraints of $D M Q_{1}$ can be transformed into pattern constraints of $D M Q_{2}$ by appending new basic Boolean pattern predicates or replacing basic Boolean pattern predicates with stronger ones.

Given two sequential pattern queries, there are four cases possible regarding pattern constrains: $D M Q_{1}$ and $D M Q_{2}$ have the same pattern constraints, $D M Q_{1}$ extends pattern constraints of $D M Q_{2}, D M Q_{2}$ extends pattern constraints of $D M Q_{1}$, or pattern constraints of $D M Q_{1}$ and $D M Q_{2}$ are not comparable.

Definition 3. We say that a data mining query $D M Q_{2}$ extends time constraints of a data mining query $D M Q_{1}$ if any of the following conditions holds: 
1. The value of the max-gap parameter in $D M Q_{2}$ is less than in $D M Q_{1}$ and both queries have the same value of the min-gap parameter, and the same value of the window-size parameter;

2. The value of the min-gap parameter in $D M Q_{2}$ is greater than in $D M Q_{1}$ and both queries have the same value of the max-gap parameter, and the same value of the window-size parameter;

3. The value of the window-size parameter in $D M Q_{2}$ is less than in $D M Q_{1}$ and both queries have the same value of the max-gap parameter, and the same value of the min-gap parameter;

4. It is possible to formulate a data mining query $D M Q_{3}$ such that $D M Q_{2}$ extends time constraints of $D M Q_{3}$ and $D M Q_{3}$ extends time constraints of $D M Q_{1}$. (The relationship of extending time constraints is transitive.)

In other words, a data mining query $D M Q_{2}$ extends time constraints of a data mining query $D M Q_{1}$ if it restricts at least one of the time parameters (max-gap, min-gap, window-size) and does not relax any time parameters.

Given two sequential pattern queries, there are four cases possible regarding time constrains: $D M Q_{1}$ and $D M Q_{2}$ have the same time constraints, $D M Q_{1}$ extends time constraints of $D M Q_{2}, D M Q_{2}$ extends time constraints of $D M Q_{1}$, or time constraints of $D M Q_{1}$ and $D M Q_{2}$ are not comparable.

Example 1. Let us consider the following three sequential pattern queries, operating on the same dataset:

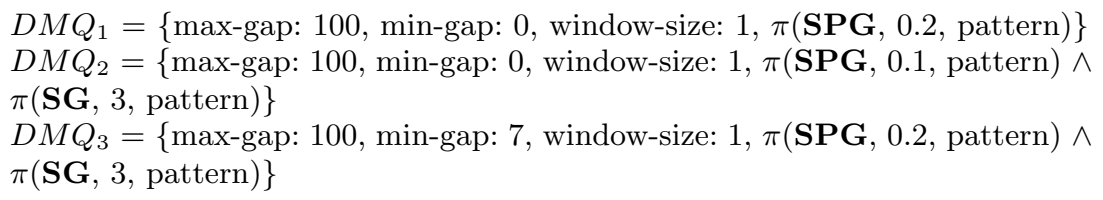

$D M Q_{3}$ extends pattern constraints of $D M Q_{1}$ and $D M Q_{2}$, while pattern constraints of $D M Q_{1}$ and $D M Q_{2}$ are not comparable. $D M Q_{3}$ extends time constraints of $D M Q_{1}$ and $D M Q_{2}$, while time constraints $D M Q_{1}$ and $D M Q_{2}$ are the same.

The two relationships defined above concern the syntax of queries, while the general inclusion and dominance relationships refer to results of queries. Below we introduce three theorems regarding dependence of relationships between results of two queries on syntactic differences between the two queries. We also introduce several lemmas on which the proofs of theorems are based. For brevity, we do not include proofs of the lemmas since they come straight from the above definitions and inherent properties of pattern and time constraints.

Lemma 1. Let $b_{1}$ and $b_{2}$ be basic Boolean pattern predicates such that $b_{2}$ is stronger than $b_{1}$. For each pattern $p$, if $p$ satisfies $b_{2}$ then $p$ satisfies $b_{1}$.

Lemma 2. Let $D M Q_{1}$ and $D M Q_{2}$ be two sequential pattern queries, operating on the same dataset and having the same time constraints. Let $p_{1}$ and $p_{2}$ denote pattern constraints of $D M Q_{1}$ and $D M Q_{2}$ respectively. If $p_{2}=p_{1} \wedge b$, where $b$ is a basic Boolean pattern predicate, then $D M Q_{1}$ includes $D M Q_{2}$. 
Lemma 3. Let $D M Q_{1}$ and $D M Q_{2}$ be two sequential pattern queries, operating on the same dataset and having the same time constraints. Let $p_{1}$ and $p_{2}$ denote pattern constraints of $D M Q_{1}$ and $D M Q_{2}$ respectively. If $p_{1}=p \wedge b_{1}$ and $p_{2}=$ $p \wedge b_{2}$, where $p$ is a conjunction of $n$ basic Boolean pattern predicates $(n \geq 0)$ and $b_{2}$ is stronger than $b_{1}$, then $D M Q_{1}$ includes $D M Q_{2}$.

Theorem 1. Let $D M Q_{1}$ and $D M Q_{2}$ be two sequential pattern queries, operating on the same dataset and having the same time constraints. If $D M Q_{2}$ extends pattern constraints of $D M Q_{1}$, then $D M Q_{1}$ includes $D M Q_{2}$.

Proof. From the Definition 2, we know that if $D M Q_{2}$ extends pattern constraints of $D M Q_{1}$, then it is possible to formulate a sequence of sequential pattern queries $D M Q_{i_{1}}, D M Q_{i_{2}}, \ldots, D M Q_{i_{n}}$ operating on the same dataset and having the same time constraints as $D M Q_{1}$ and $D M Q_{2}$, such that $D M Q_{i_{1}}=D M Q_{1}$ and $D M Q_{i_{n}}=D M Q_{2}$, and for $j=2 . . n$ one of the following conditions holds:

1. pattern constraints of $D M Q_{i_{j-1}}$ have a form of a conjunction of $n$ basic Boolean pattern predicates, pattern constraints of $D M Q_{i_{j}}$ have a form of a conjunction of $n+1$ basic Boolean pattern predicates $(n \geq 0)$, and each basic Boolean pattern predicates in $D M Q_{i_{j-1}}$ also appears in $D M Q_{i_{j}}$;

2. $D M Q_{i_{j-1}}$ and $D M Q_{i_{j}}$ have pattern constraints $p_{1}$ and $p_{2}$ respectively, where $p_{1}$ and $p_{2}$ are conjunction of $n$ basic Boolean pattern predicates $(n \geq 1)$, $p_{1}=p \wedge b_{1}, p_{2}=p \wedge b_{2}$ ( $p$ is a conjunction of $n-1$ basic Boolean pattern predicates), and $b_{2}$ is stronger than $b_{1}$.

From the Lemmas 2 and 3 and the transitivity property of the inclusion relationship, we have $D M Q_{1}$ includes $D M Q_{2}$.

Lemma 4. Let $D M Q_{1}$ and $D M Q_{2}$ be two sequential pattern queries, operating on the same dataset and having the same pattern constraints. Let max $_{1}$, min $_{1}$, and win $_{1}$ denote values of max-gap, min-gap, and window-size parameters of $D M Q_{1}$, and $\max _{2}, \min _{2}$, and win 2 values of max-gap, min-gap, and windowsize parameters of $\mathrm{DMQ}_{2}$. If one of the following conditions holds:

1. $\max _{2}<\max _{1}$ and $\min _{2}=\min _{1}$ and $\operatorname{win}_{2}=\operatorname{win}_{1}$,

2. $\min _{2}>\min _{1}$ and $\max _{2}=\max _{1}$ and $\operatorname{win}_{2}=\operatorname{win}_{1}$,

3. win $_{2}<$ win $_{1}$ and $\max _{2}=\max _{1}$ and $\min _{2}=\min _{1}$

then $D M Q_{1}$ dominates $D M Q_{2}$.

Theorem 2. Let $D M Q_{1}$ and $D M Q_{2}$ be two sequential pattern queries, operating on the same dataset and having the same pattern constraints. If $D M Q_{2}$ extends time constraints of $D M Q_{1}$, then $D M Q_{1}$ dominates $D M Q_{2}$.

Proof. Let $\max _{1}, \min _{1}$, and $w_{i n}$ denote values of max-gap, min-gap, and window-size parameters of $D M Q_{1}$, and $\max _{2}, \min _{2}$, and $w_{i n}$ values of maxgap, min-gap, and window-size parameters of $D M Q_{2}$. Since $D M Q_{2}$ extends time constraints of $D M Q_{1}$, we have: $\operatorname{win}_{2} \leq$ win $_{1}, \max _{2} \leq \max _{1}$ and $\min _{2} \geq \min _{1}$. Let $D M Q_{3}$ and $D M Q_{4}$ be sequential pattern queries operating on the same 
dataset and having the same pattern constraints as $D M Q_{1}$ and $D M Q_{2}$, Let the values of max-gap, min-gap, and window-size parameters be $\max _{2}, \min _{1}$, and $w_{i n}$ in case of $D M Q_{3}$, and $\max _{2}, \min _{2}$, and $w_{i n}$ in case of $D M Q_{4}$. Thus, from the Lemma $4, D M Q_{1}$ dominates $D M Q_{3}, D M Q_{3}$ dominates $D M Q_{4}$, and $D M Q_{4}$ dominates $D M Q_{2}$ (in fact, in each of the three cases equivalence is possible but equivalence is a particular case of dominance). Since the dominance relationship is transitive, $D M Q_{1}$ dominates $D M Q_{2}$.

Theorem 3. Let $D M Q_{1}$ and $D M Q_{2}$ be two sequential pattern queries, operating on the same dataset. If $D M Q_{2}$ extends pattern constraints of $D M Q_{1}$ and $D M Q_{2}$ extends time constraints of $D M Q_{1}$, then $D M Q_{1}$ dominates $D M Q_{2}$.

Proof. Let $D M Q_{3}$ be a sequential pattern query operating on the same dataset as $D M Q_{1}$ and $D M Q_{2}$, having pattern constraints of $D M Q_{1}$ and time constraints of $D M Q_{2}$. Thus, $D M Q_{2}$ extends pattern constraints of $D M Q_{3}$ and $D M Q_{3}$ extends time constraints of $D M Q_{1}$. From the Theorems 1 and 2 we have: $D M Q_{1}$ dominates $D M Q_{3}$ and $D M Q_{3}$ includes $D M Q_{2}$. Since inclusion is a particular case of dominance and the dominance relationship is transitive, $D M Q_{1}$ dominates $D M Q_{2}$.

\section{Algorithms for Efficient Sequential Pattern Query Processing in the Presence of Materialized Results of Previous Queries}

Given a sequential pattern query $D M Q$ and materialized results of a sequential pattern query $D M Q_{V}$, in the general case, even if $D M Q_{V}$ and $D M Q$ operate on the same dataset but differ in pattern and time constraints, it is not possible to answer $D M Q$ without running a sequential pattern mining algorithm. However, there are four particular cases where $D M Q$ can be answered efficiently using the materialized results of $D M Q_{V}$ since they correspond to equivalence, inclusion, and dominance relationships between $D M Q_{V}$ and $D M Q$. These cases are listed below:

1. If $D M Q_{V}$ and $D M Q$ have the same pattern and time constraints, then the results of $D M Q$ are equal to the results of $D M Q_{V}$ (the two queries are equivalent since they are identical);

2. If $D M Q_{V}$ and $D M Q$ have the same time constraints and $D M Q$ extends pattern constraints of $D M Q_{V}$, then $D M Q$ can be answered by filtering out the patterns returned by $D M Q_{V}$ not satisfying pattern constraints of $D M Q$ ( $D M Q_{V}$ includes $D M Q$ according to the Theorem 1);

3. If $D M Q_{V}$ and $D M Q$ have the same pattern constraints and $D M Q$ extends time constraints of $D M Q_{V}$, then $D M Q$ can be answered by evaluating the support of the patterns returned by $D M Q_{V}$ using the time constraints of $D M Q$, and filtering out patterns not satisfying the minimum support threshold of $D M Q$. $\left(D M Q_{V}\right.$ dominates $D M Q$ according to the Theorem 2); 
4. If $D M Q$ extends pattern constraints of $D M Q_{V}$ and $D M Q$ extends time constraints of $D M Q_{V}$, then $D M Q$ can be answered by evaluating the support of the patterns returned by $D M Q_{V}$ using the time constraints of $D M Q$, and filtering out patterns not satisfying the pattern constraints of $D M Q$. $\left(D M Q_{V}\right.$ dominates $D M Q$ according to the Theorem 3).

Answering the query in the first case (the case of equivalence) is trivial, therefore we concentrate on details concerning inclusion and dominance relationships.

For the second case we propose an algorithm that performs one sequential scan of the materialized patterns, processing one pattern at a time (main memory requirements are minimal). Each pattern is tested if it satisfies these basic Boolean pattern predicates from the pattern constraints of $D M Q$ that were not in $D M Q_{V}$. All the basic Boolean pattern predicates of $D M Q$ that were in $D M Q_{V}$ must be satisfied by all the materialized patterns since pattern constraints in our model have the form of a conjunction of basic predicates. The algorithm for the second case is presented below.

Algorithm 1 Answering a sequential pattern query in case of inclusion due to extending pattern constraints (Result Filtering)

Input: A sequential pattern query issued by a user $(D M Q)$ and results of a sequential pattern query $D M Q_{V}$ including $D M Q$.

Output: The results of $D M Q$.

Method:

begin

Answer $=$ results of $D M Q_{V}$;

for each $p \in$ results of $D M Q_{V}$ do

begin

for each basic Boolean pattern predicate $b$ such that

$b$ is in pattern constraints of $D M Q$ and

$b$ is not in pattern constraints of $D M Q_{V}$ do

begin

if not ( $p$ satisfies $b)$ then

Answer $=$ Answer $\backslash\{p\}$

break;

end if:

end;

end;

output Answer;

end.

For the third and fourth cases we propose one uniform algorithm (both cases result in the dominance relationship). Conceptually, the algorithm has to scan the source dataset once in order to re-evaluate the support of materialized patterns and then prune the patterns that do not satisfy pattern constraints of $D M Q$. However, for the fourth case, we apply one optimization to reduce the cost of the support re-evaluation phase that is proportional to the number of patterns 
to be verified. Before scanning the source dataset, we filter out patterns that do not satisfy pattern constraints of $D M Q$ using Algorithm 1. After the scan of the dataset, we only test the predicate representing the minimum support threshold (the only one that for a given pattern could by true before the support re-evaluation, and false after that operation). The effects of this optimization will be discussed in the next section.

During the support re-evaluation phase, when testing whether a currently processed data-sequence contains a given pattern, all time constraints of $D M Q$ have to be taken into account, even if only one of them has been restricted compared to $D M Q_{V}$. This is motivated by the observation that a given pattern may occur several times in a given data-sequence. As a result, if we checked only one of the time constraints, we might find a different occurrence satisfying the constraint than the occurrence previously found as valid with respect to the other two time constraints.

The algorithm in the form presented below assumes that the set of materialized patterns supporting pattern constraints of $D M Q$ fits into main memory. If this is not the case, the set of materialized patterns has to be partitioned into portions that fit into main memory and the algorithm has to be run on each of the partitions.

Algorithm 2 Answering a sequential pattern query in case of dominance due to extending time constraints (Result Verification)

Input: A sequential pattern query issued by a user $(D M Q)$, a collection of data-sequences $D$, and results of a sequential pattern query $D M Q_{V}$ dominating $D M Q$.

Output: The results of $D M Q$.

Method:

begin

if $D M Q$ extends pattern constraints of $D M Q_{V}$ then

Answer $=$ patterns in results of $D M Q_{V}$ satisfying

pattern constraints of $D M Q ; / *$ Algorithm $1 * /$

else Answer $=$ results of $D M Q_{V}$;

end if;

scan $D$ once evaluating the support of patterns

in Answer using time constraints of $D M Q$;

for each $p \in$ Answer do

begin

if $p$ exceeds the minimum support threshold of $D M Q$

then output $p$; end if; end;

end.

Having provided sequential pattern query processing algorithms for the cases leading to equivalence, inclusion and dominance relationships, we have to address situations where for a given query issued by a user $(D M Q)$, there are 
many materialized query results that could be used to answer the query without running a complete data mining algorithm. In general, the set of applicable materialized query results consists of results of queries equivalent to $D M Q$, including $D M Q$, and dominating $D M Q$. It is clear that in the first place the data mining system should look for a query identical to $D M Q$ (the case of equivalence) since in that case the results of $D M Q$ are directly available. Then, the system should look for query results that could be used by Algorithm 1 (returned by a query $D M Q_{V}$ having the same time constraints as $D M Q$, such that $D M Q$ extends pattern constraints of $D M Q_{V}$ ). If no query satisfying the above criteria could be found, the system should try to find query results that could be used by Algorithm 2 (returned by a query $D M Q_{V}$, such that $D M Q$ extends time constraints of $D M Q_{V}$ and either $D M Q_{V}$ and $D M Q$ have the same pattern constraints or $D M Q$ extends pattern constraints of $D M Q_{V}$ ). Finally, if again no appropriate query criteria could be found, a complete data mining algorithm has to be run.

We believe that in majority of cases Algorithm 1 will be more efficient than Algorithm 2 since the former requires one scan of the pattern set and no scan of the source dataset, while the latter scans the source dataset once and during this scan for each data-sequence processes all the patterns. However, it has to be noted that in certain cases application of Algorithm 2 may be more efficient than application of Algorithm 1 (for example, if the source dataset and the materialized set of patterns to be used by Algorithm 2 are extremely small, whereas the materialized pattern set to be used by Algorithm 1 is huge).

The final issue that has to be addressed is the selection of the materialized query results to be used by Algorithms 1 and 2 if there is more than one query including or dominating the query to be answered. We observe that it is not possible to provide selection criteria always leading to the minimal processing time, because the processing time depends not only on the syntax of the queries but also on the contents of the source dataset. Therefore, we decide to optimize the space requirements by choosing the materialized pattern set of the smallest size. We believe that this solution will also lead to minimal processing time in many situations, since smaller size of the pattern set leads to the smaller number or size of patterns that have to be filtered or verified against the database. It is not guaranteed, however, since the processing time is affected also by the number of predicates that have to be tested for each pattern, which depends on the pattern structure (subsequent predicates are tested until one of them is found to be false).

Example 2. Let us consider the following three queries discovering sequential patterns from the same dataset:

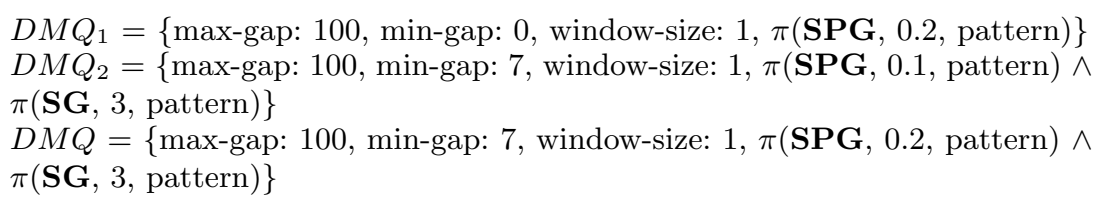

Let us assume that results of $D M Q_{1}$ and $D M Q_{2}$ are stored in cache, and $D M Q$ is the query to be answered. Since neither $D M Q_{1}$ nor $D M Q_{2}$ is identical to 
$D M Q$, the data mining system would choose to answer $D M Q$ using Algorithm 1 exploiting cached results of $D M Q_{2}$ (returning those patterns from the results of $D M Q_{2}$ that exceed the minimum support threshold of 0.2). If results of $D M Q_{2}$ were not available, the system would answer $D M Q$ using Algorithm 2 exploiting the results of $D M Q_{1}$ (selecting patterns from the results of $D M Q_{1}$ whose size is greater than 3 , re-evaluating their support in one scan of the source dataset using max-gap of 100, min-gap of 7 , and window-size of 1 , and returning those patterns that exceed the minimum support threshold of 0.2 after support re-evaluation).

\section{Experimental Results}

In order to evaluate performance gains offered by our sequential pattern query processing algorithms, we performed several experiments on a synthetic dataset generated by means of the GEN generator from the Quest project [1]. We treated transaction identifiers generated by GEN as transaction times. Thus, the time gap between two adjacent elements of each data-sequence was always equal to one time unit. The dataset used in the experiments consisted of 1000 datasequences. GEN parameter values were chosen so that for the minimum support thresholds used in queries there were a reasonable number of sequential patterns varying in size and length to be discovered.

In the first step we materialized the results of the query discovering all sequential patterns whose support was above $0.5 \%$ using max-gap of 1000, mingap of 0 , and window-size of 1 . The materialized set of patterns consisted of about 3500 sequential patterns. Next, we tested several queries adding additional pattern constraints (concerning pattern support, size, length, or contents) and restricting time constraints. For each query, we compared execution times of our algorithms exploiting materialized patterns and the GSP algorithm with the post-processing pattern filtering phase. For the queries included by the materialized query, Algorithm 1 was on average more than 400 times faster than GSP. For the queries dominated by the materialized query, Algorithm 2 was used, and its processing time was on average more than 100 times shorter than in case of GSP. We also tested the effects of our optimization used in case of queries extending both pattern and time constraints of the materialized query (filtering out patterns that do not satisfy pattern constraints before re-evaluating the support of materialized patterns). Experiments show that the optimization reduces processing time by about $33 \%$.

\section{Concluding Remarks}

We proved experimentally that our sequential pattern query processing schemes can reduce processing time by several orders of magnitude when materialized results of previous queries are available. However, theoretically it is possible to imagine situations, where a complete mining algorithm could be more efficient than our techniques. While we believe that in typical situations our methods 
should outperform mining algorithms, in the future we plan to focus on costbased optimization of sequential pattern queries (and data mining queries in general) using certain statistics of the source dataset in order to choose an optimal query execution plan.

In the paper, we did not discuss cache management schemes, which could certainly influence the overall performance of the system. We believe that general purpose cache management algorithms could be used, possibly with simple optimizations such as removing included or dominated queries first, and not materializing results of queries equivalent to queries whose results are already in cache.

\section{References}

1. Agrawal R., Mehta M., Shafer J., Srikant R., Arning A., Bollinger T.: The Quest Data Mining System. Proc. of the 2nd KDD Conference (1996)

2. Agrawal R., Srikant R.: Mining Sequential Patterns. Proc. of the 11th ICDE Conf. (1995)

3. Baralis E., Psaila G.: Incremental Refinement of Mining Queries. Proc. of the 1st DaWaK Conference (1999)

4. Han J., Lakshmanan L., Ng R.: Constraint-Based Multidimensional Data Mining. IEEE Computer, Vol. 32, No. 8 (1999)

5. Han J., Pei J., Mortazavi-Asl B., Chen Q., Dayal U., Hsu M-C.: FreeSpan: Frequent Pattern-Projected Sequential Pattern Mining. Proc. of the 6th KDD Conference (2000)

6. Imielinski T., Mannila H.: A Database Perspective on Knowledge Discovery. Communications of the ACM, Vol. 39, No. 11 (1996)

7. Nag B., Deshpande P.M., DeWitt D.J.: Using a Knowledge Cache for Interactive Discovery of Association Rules. Proc. of the 5th KDD Conference (1999)

8. Parthasarathy S., Zaki M.J., Ogihara M., Dwarkadas S.: Incremental and Interactive Sequence Mining. Proc. of the 8th CIKM Conference (1999)

9. Pei J., Han J., Mortazavi-Asl B., Pinto H., Chen Q., Dayal U., Hsu M-C.: PrefixSpan: Mining Sequential Patterns Efficiently by Prefix-Projected Pattern Growth. Proc. of the 17th ICDE Conference (2001)

10. Srikant R., Agrawal R.: Mining Sequential Patterns: Generalizations and Performance Improvements. Proc. of the 5th EDBT Conference (1996) 